\title{
The Growth of the Stellar Seeds of Supermassive Black Holes
}

\author{
Jarrett L. Johnson ${ }^{* \dagger}$, Bhaskar Agarwal ${ }^{\dagger}$, Daniel J. Whalen*,*, Claudio \\ Dalla Vecchia ${ }^{\dagger}$, Christopher L. Fryer*, Sadegh Khochfar ${ }^{\dagger}, \mathrm{Hui} \mathrm{Li}^{*}$ and \\ Mario Livio ${ }^{\ddagger}$ \\ *Los Alamos National Laboratory, Los Alamos, NM 87545 \\ ${ }^{\dagger}$ Max-Planck-Institut für extraterrestrische Physik, 85748 Garching, Germany \\ ${ }^{*}$ Carnegie Mellon University, Pittsburgh, PA 15213 \\ ${ }^{\ddagger}$ Space Telescope Science Institute, Baltimore, MD 21218
}

\begin{abstract}
One of the most promising explanations for the origin of the billion solar mass black holes (BHs) inferred to power quasars at redshifts $z \geq 6$ is that supermassive stars (SMSs) with masses $\geq 10^{4} \mathrm{M}_{\odot}$ collapse to form the seed BHs from which they grow. Here we review recent theoretical advances which provide support for this scenario. Firstly, given sufficiently high accretion rates of gas into the cores of primordial protogalaxies, it appears that neither the high energy radiation emitted from the stellar surface nor the limited lifetime of SMSs can prevent their growth to masses of up to $\geq 10^{5} \mathrm{M}_{\odot}$. Secondly, recent cosmological simulations suggest that the high fluxes of molecule-dissociating radiation which may be required in order to achieve such high accretion rates may be more common in the early universe than previously thought. We conclude that the majority of supermassive BHs may originate from SMSs at high redshifts.
\end{abstract}

Keywords: black holes, supermassive stars, high redshift, galaxy formation

PACS: 97.60.Lf, 97.10.Bt, 97.10.Gz, 95.30.Ft, 95.30.Lz, 95.30.Jx

\section{INTRODUCTION: THE RAPID GROWTH OF BLACK HOLES}

There is growing observational evidence that the formation and growth of black holes (BHs) in the earliest galaxies was, in many cases, very rapid. Particularly strong constraints on the pace at which early BHs grow comes from observations of quasars at $z$ $\geq 6$ which are inferred to be powered by accretion onto BHs with masses $\geq 10^{9} \mathrm{M}_{\odot}$ (e.g. Mortlock et al. 2011). Given the complexity of the formation process of the early galaxies which harbored the first BHs, the intense radiative feedback associated with their accretion, and the large amount of material that they must accrete in such a short time, it is a challenge to understand the origin of these objects (e.g. Volonteri 2010).

One promising explanation for how BHs grow to supermassive scales by the time the universe is only a few percent of its present age is that the $\mathrm{BH}$ seeds from which they grew were initially relatively massive themselves. This is especially true if the rate at which BHs can grow is limited by the radiation emitted in the accretion process (e.g. the Eddington limit), as in this case smaller BHs cannot grow as quickly as larger ones. As such seed BHs were likely born from the collapse of primordial stars, this raises the question of how massive such stars can become. As we discuss below, there is growing theoretical evidence for a population of supermassive stars (SMSs), with masses $\geq 10^{4}$, that are strong candidates for the seeds of SMBHs. 


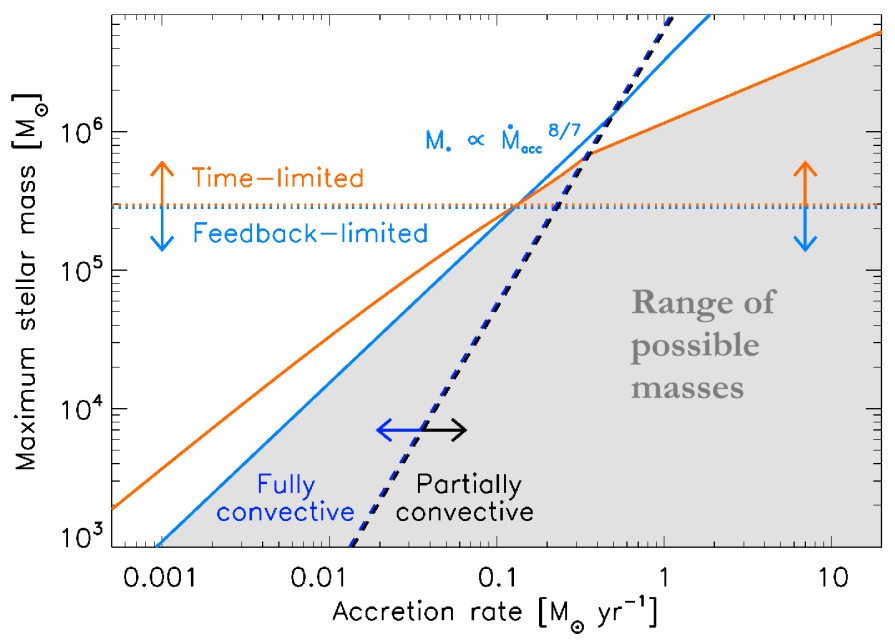

FIGURE 1. Maximum mass $M_{*}$ to which a star can grow at a constant accretion rate $\dot{M}_{\text {acc }}$. Above the maximum mass $M_{*} \propto \dot{M}_{\text {acc }}^{8 / 7}$ (blue line), radiative feedback shuts off accretion because the $\mathrm{H}$ II region of the star breaks out and prevents gas infall. For accretion rates $\dot{M}_{\text {acc }} \geq 10^{-1} \mathrm{M}_{\odot} \mathrm{yr}^{-1}$, the maximum mass is set instead by the lifetime of the star (red line). From Johnson et al. (2012a).

\section{THE FORMATION OF SUPERMASSIVE STARS}

From simple theoretical arguments, the characteristic mass of stars is thought to increase with the temperature of the gas from which they form. As the first, Population (Pop) III stars formed from gas cooled only by the relatively inefficient coolant molecular hydrogen $\left(\mathrm{H}_{2}\right)$ to $\simeq 200 \mathrm{~K}$ (an order of magnitude higher than that of metal-enriched, star-forming gas today), they were likely relatively massive (e.g. Bromm \& Larson 2004). Furthermore, in regions of the early universe where the formation of $\mathrm{H}_{2}$ was suppressed, for example by a large flux of $\mathrm{H}_{2}$-dissociating stellar radiation, the gas could only cool via atomic hydrogen down to $\simeq 10^{4} \mathrm{~K}$ (e.g. Bromm \& Loeb 2003; Regan \& Haehnelt 2009). Cosmological simulations following the collapse of such hot primordial gas in protogalaxies suggest that the objects that form accrete at rates $\geq 0.1 \mathrm{M}_{\odot} \mathrm{yr}^{-1}$ (Wise et al. 2008; Shang et al. 2010) and may become SMSs with final masses of $\geq 10^{5}$.

This picture, however, is complicated by the strong ionizing radiation that such SMSs would emit once they enter the main sequence; at some point, the increasing intensity of the radiative feedback from the star should halt the flow of gas onto its surface and limit its growth (e.g. Johnson et al. 2012a). A second effect which may limit the growth of SMSs is their short lifetime; once their nuclear fuel is exhausted, they cannot grow any longer and will collapse to form the seed BHs discussed above (e.g. Begelman 2010). As shown in Fig. 1, recent analytical calculations suggest that the first effect limits the growth of SMSs which accrete at rates $\leq 0.1 \mathrm{M}_{\odot} \mathrm{yr}^{-1}$ to $\sim 10^{5} \mathrm{M}_{\odot}$, but that the most rapidly accreting SMSs will only be limited by the second effect and may grow to larger masses (Johnson et al. 2012a). Thus, given the extremely high accretion rates rates found in cosmological simulations, it appears that little can prevent the growth of SMSs up to $\sim 10^{5}-10^{6} \mathrm{M}_{\odot}$, and their subsequent collapse to seed BHs of similar masses. 

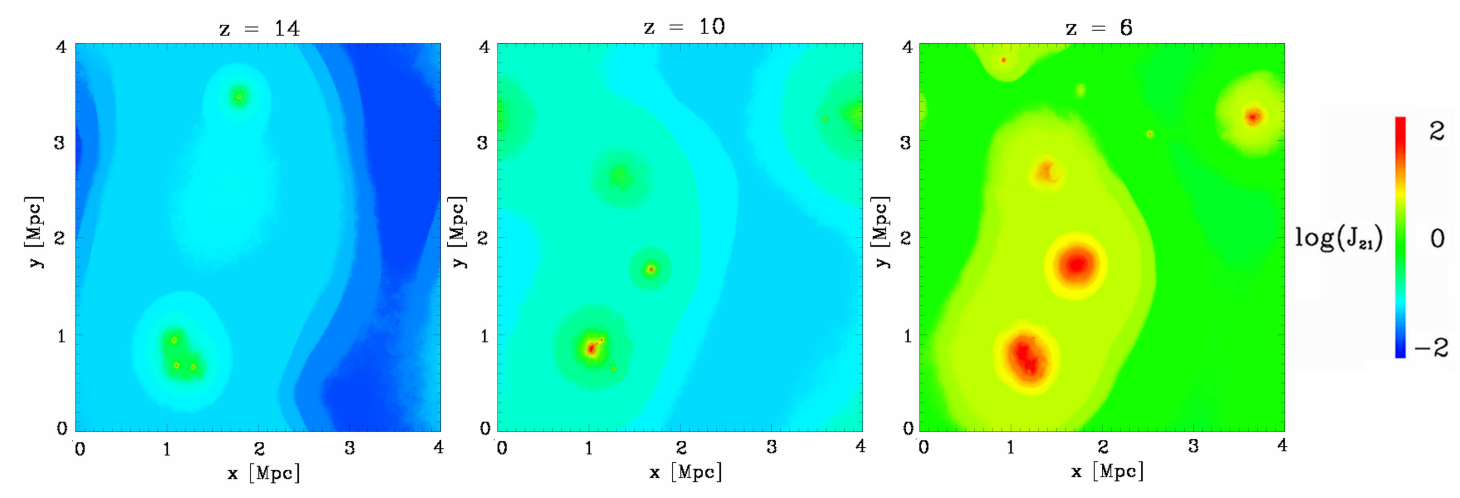

FIGURE 2. The $\mathrm{H}_{2}$-dissociating flux $\left(J_{21}\right)$ generated by Pop II and III stars in a simulated cosmological volume, at redshifts $z=14$ (left), 10 (middle), and 6 (right). SMSs are expected to form in the cores of massive primordial halos that are exposed to fluxes of at least $J_{21} \simeq 30-10^{3}$. From Johnson et al. (2012b).

\section{THE UBIQUITY OF SUPERMASSIVE STELLAR SEEDS}

As mentioned above, SMSs may only form in regions of the early universe where an especially high flux of $\mathrm{H}_{2}$-dissociating radiation is produced, mostly likely by stellar sources. Expressed in units of $10^{-21} \mathrm{erg} \mathrm{s}^{-1} \mathrm{~cm}^{-2} \mathrm{~Hz}^{-1} \mathrm{sr}^{-1}$, the flux required has been estimated to be in the range $J_{21} \sim 30-10^{3}$, with higher or lower values applying depending on the mass and metallicity of the stars producing the flux (e.g. Shang et al. 2010). While these fluxes may be well above the average expected to pervade the universe at $z \geq 6$ (but see Petri et al. 2012), recent N-body cosmological simulations suggest that in clustered regions they can be generated locally by early metal-enriched stellar clusters (Agarwal et al. 2012). Indeed, these results suggest that the conditions for SMS formation may be much more common in the early universe than previously thought, perhaps leading to the majority of SMBHs being seeded by SMSs. As shown in Fig. 2, recent cosmological hydrodynamics simulations accounting explicitly for metal enrichment, Pop II and III star formation, and the locally- and globally-generated $\mathrm{H}_{2}$ dissociating radiation have corroborated this basic finding (Johnson et al. 2012b).

\section{SUMMARY AND OUTLOOK}

The two main conclusions of the work presented here are the following:

- Given the high accretion rates of gas into the cores of protogalaxies found from cosmological simulations, it appears that neither radiative feedback nor the limited lifetime of SMSs prevents their growth up to $\sim 10^{5}-10^{6} \mathrm{M}_{\odot}$ (Johnson et al. 2012a).

- The conditions under which such high accretion rates can be generated may be much more common than previously assumed, leading perhaps to the seeding of a majority of SMBHs by SMSs (Agarwal et al. 2012; Johnson et al. 2012b).

Ultimately, these findings will have to be verified by observations, such as those scheduled to be conducted by the James Webb Space Telecope (JWST). As Fig. 3 shows, 


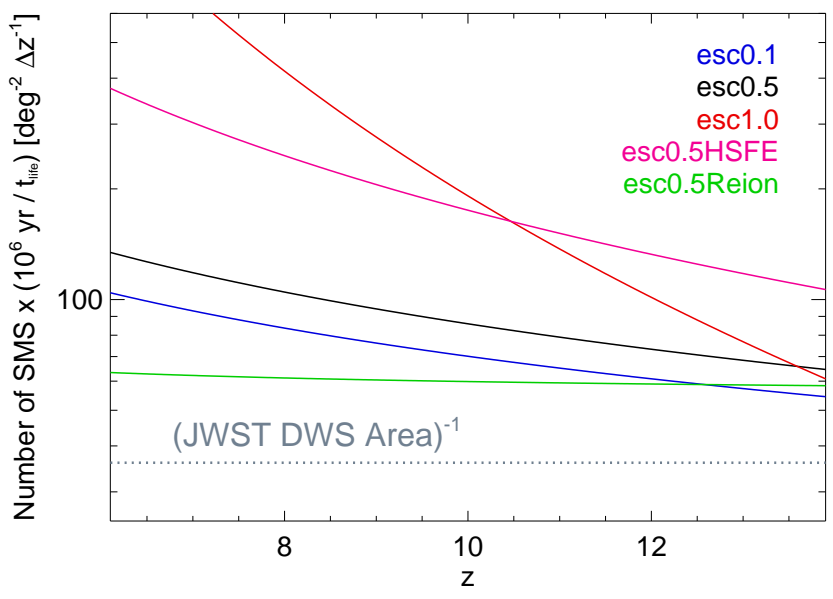

FIGURE 3. The number of supermassive stellar progenitors of direct collapse black holes, as observed on the sky per square degree per redshift interval $(\Delta z)$, as a function of redshift $z$. The gray dotted line shows the number of SMSs that must be present for at least one per redshift interval $(\Delta z=1)$ to appear in the field of view of the Deep-Wide Survey planned for the JWST. The survey should be large enough for up to of the order of 10 SMSs to lie within the field of view. From Agarwal et al. (2012).

Agarwal et al. (2012) find that SMSs may form at sufficiently high rates for of the order of 10 to appear in the deep survey fields of the JWST. Also, due to the high accretion rates of primordial gas onto these objects, they are predicted to have unique observable signatures. Among these are strong Balmer series and He II $\lambda 1640$ emission, as well as comparatively weak Ly $\alpha$ emission (Johnson et al. 2012a).

\section{ACKNOWLEDGMENTS}

JLJ thanks the organizers of First Stars IV for the opportunity to present this work, much of which was generously supported by the LANL LDRD program.

\section{REFERENCES}

1. B. Agarwal, S. Khochfar, J. L. Johnson, E. Neistein, C. Dalla Vecchia, M. Livio, Mon. Not. of the Royal Astron. Soc. submitted, arXiv:1205.6464 (2012).

2. M. C. Begelman, Mon. Not. of the Royal Astron. Soc. 402, 673-681 (2010).

3. V. Bromm, A. Loeb, Astrophys. J. 596, 34-46 (2003).

4. V. Bromm, R. Larson, Ann. Rev. of Astron. \& Astophys. 42, 79-118 (2004).

5. J. L. Johnson, D. J. Whalen, C. L. Fryer, H. Li, Astrophys. J. 750, 66-77 (2012a).

6. J. L. Johnson, C. Dalla Vecchia, S. Khochfar, Mon. Not. of the Royal Astron. Soc. in press (2012b).

7. D. J. Mortlock, et al., Nature 474, 616-619 (2009).

8. A. Petri, A. Ferrara, R. Salvaterra, Mon. Not. of the Royal Astron. Soc. 422, 1690-1699 (2012).

9. J. A. Regan, M. G. Haehnelt, Mon. Not. of the Royal Astron. Soc. 396, 343-353 (2009).

10. C. Shang, G. L. Bryan, Z. Haiman, Mon. Not. of the Royal Astron. Soc. 402, 1249-1262 (2010).

11. M. Volonteri, Astron. \& Astrophys. Rev. 18, 279-315 (2010).

12. J. H. Wise, M. J. Turk, T. Abel, Astrophys. J. 682, 745-757 (2008). 